J. Biosci., Vol. 22, Number 1, January 1997, pp 33-45. (C) Printed in India.

\title{
In vivo clearance of Japanese encephalitis virus by adoptively transferred virus specific cytotoxic $T$ lymphocytes
}

\author{
A DESAI*, K MURALI-KRISHNA**, B RAMIREDDY, \\ V RAVI* and R MANJUNATH ${ }^{\dagger}$ \\ Department of Biochemistry, Indian Institute of Science, Bangalore 560 012, India \\ *Department of Neurovirology, National Institute for Mental Health and Neuro Sciences, \\ Bangalore 560 029, India \\ ** Department of Microbiology and Immunology, Emory University School of Medicine, \\ Atlanta, Georgia 30322, USA
}

MS received 29 February 1996; revised 16 July 1996

\begin{abstract}
Japanese encephalitis virus (JEV) is a positive stranded RNA virus that belongs to the flavivirus group. JEV infection damages the central nervous system (CNS) and is one of the main causative agents of acute encephalitis. H-2 restricted virus-specific cytotoxic T lymphocytes (CTL) have been generated specifically against JEV in our laboratory and these CTL have been shown to protect mice against lethal challenge with JEV. Virus replication was found to be inhibited in the brains of animals that were adoptively transferred with JEV specific CTL as revealed by immunohistological staining as well as viral plaque assays. We further show that virus specific CTL could be recovered from such protected mice as long as 45 days after adoptive transfer.
\end{abstract}

Keywords. Cytotoxic T lymphocytes; adoptive transfer; clearance in vivo; Japanese encephalitis virus.

\section{Introduction}

Japanese encephalitis virus (JEV) is a positive stranded RNA virus whose epidemiological and structural aspects have been extensively reviewed (Chambers et al 1990; Monath et al 1986). It is a flavivirus (Westaway et al 1985) which causes acute encephalitis associated with damage to central nervous system (CNS) (Oyanagi et al 1969). JEV has been declared endemic in many parts of India with sporadic epidemics occurring periodically in West Bengal, Uttar pradesh, Karnataka, Haryana and other parts of India. Passive administration of anti-JEV antibodies have been shown to confer protection against JEV in mice (Kimura - Kuroda and Yasui 1988). However, the observation that adoptive transfer of immune T-cells can protect mice from a subsequent challenge with JEV (Mathur et al 1983) and intracerebral challenge experiments with nude mice (Lad et al 1993) suggests that T-cells also play a role in host-defense against JEV. These observations emphasise the importance of studies on the cell-mediated immune responses against these viruses. MHC-restricted cytotoxic $\mathrm{T}$ lymphocytes (CTL) have been generated that have the ability to lyse West Nile virus (WNV)-infected and dengue virus-infected cells (Hill et al 1955; Rothman et al 1993). CTL have been shown to play a crucial role in recovery from several viral infections such as influenza, lymphocytic choriomeningitis virus, rabies and others (Cossins et al

${ }^{\dagger}$ Corresponding author (Fax, 91-80-3341683; Email, mjnhm@biochem. iisc, ernet.in) 
1993; Joly et al 1991; Kawano et al 1990). We have previously demonstrated that Lyt $2.2 \mathrm{CTL}$ can be raised against JEV-infected targets in Balb/c mice. Both $\mathrm{H}-2 \mathrm{~K}^{d} \mathrm{D}^{d} / \mathrm{L}^{d}$ and $\mathrm{H}-2 \mathrm{k}^{k} \mathrm{D}^{d /} \mathrm{L}^{d}$ JEV infected targets were lysed (Murali-Krishna et al 1994). In this report we demonstrate that intracerebral administration of the anti-JEV CTL generated can protect mice from lethal challenge with $1 \mathrm{EV}$ by blocking the growth of virus in the brain. We further show that anti viral CTL can be recovered from the spleen of adoptively transferred mice 45 days after the transfer.

\section{Materials and methods}

\section{$2.1 \quad$ Mice}

Balb/c $\left(H-2^{d}\right)$ mice were obtained from the Institute colony and 6-8 week old mice were used for all studies.

\subsection{Virus growth and infection of cell lines}

JEV strain P20778, an isolate from human brain (Kedarnath et al 1986) was routinely grown in C6/36 cells that were cultured to confluency in MEM supplemented with $10 \%$ FBS (GIBCO, Grand Island, New York, USA). Infected C6/36 cell culture supernatants were used as the source of virus in all our studies. The viral titration was carried out by plaguing on porcine kidney (PS) cells as described (Gould and Clegg 1985) with modifications. Infection of different cell lines was carried out as described previously (MuraliKrishna et al 1995). Virus growth and infection was monitored by measuring the release of infectious viral particles into the culture supernatants as above and by immunofluorescence staining of cells using monoclonal antibodies specific to the $1 \mathrm{EV}$ envelope $(\mathrm{E})$ antigen.

\subsection{Virus titration in infected mouse brains}

JEV injected adult mice were killed by ether anaesthesia, the brains were dissected out and frozen at $-70^{\circ} \mathrm{C}$. A $10 \%$ weight/volume homogenate was obtained in PBS and centrifuged at 10,000 $\mathrm{g}$ for $10 \mathrm{~min}$ and the sterile filtered tissue homogenate was titrated by plaguing on PS cells.

\section{$2.4 \quad$ Stimulator and target cells}

P388D1, P815 (DBA/2 mouse origin, H-2 ${ }^{d}$ ); YAC-1 and L929 (C3H mouse origin, H-2 ${ }^{k}$ ) were cultured at $37^{\circ} \mathrm{C}$ and $5 \% \mathrm{CO}_{2}$ atmosphere in RPMI 1640 medium supplemented with antibiotics and 5\% FBS (GIBCO). All cell lines were obtained from the National Facility for Animal Tissue and Cell Culture Collections (NFATCC, Pune).

\subsection{Generation of anti-JEV CTL}

Eight week old male Balb/c mice were generally immunized intraperitoneally (ip) twice with $3 \times 10^{6} \mathrm{JEV}$-infected PS cells followed by $1 \times 10^{7} 1 \mathrm{EV}$-infected Sp 2/0 cells at 3 day 
intervals on day 0 , day 3 and day 6 respectively. Mice that were protected during adoptive transfer studies were also used for generation of CTL. These were generally $6-7$ months old when immunized. Animals were killed on day 10 and $1 \times 10^{7}$ primed splenocytes (responder cells) were cultured in vitro in 24-well Linbro plates (Costar, USA) with $2 \times 10^{5}$ virus-infected syngeneic stimulator cells. Effector cells were generated at $37^{\circ} \mathrm{C}$ in a $5 \% \mathrm{CO}_{2}$ atmosphere by in vitro culture in RPMI 1640 medium supplemented with antibiotics, $5 \times 10^{4} \mathrm{M} 2$-mercaptoethanol, $0 \cdot 32 \mathrm{mg} / \mathrm{ml}$ L-glutamine, $01 \mathrm{mg} / \mathrm{ml}$ nonessential amino acids, $0.12 \mathrm{mg} / \mathrm{ml}$ sodium pyruvate and $5 \%$ FBS. The number of virus particles used to infect various cell lines and the times of harvest after $1 \mathrm{EV}$ infection were standardized to ensure maximum possible infection at optimal cell viabilities (Murali-Krishna et al 1995).

\subsection{CTL assays and target cell preparations}

Varying numbers of effector cells generated in mixed lymphocyte cultures (MLC) were incubated with $2 \times 10^{4},{ }^{51} \mathrm{Cr}$-labelled virus-infected or uninfected cells for $5 \mathrm{~h}$ at $37^{\circ} \mathrm{C}$ after briefly centrifuging them together in 96 well $\mathrm{V}$-bottom plates at $100 \mathrm{~g}$ for $4 \mathrm{~min}$. Target cells were prepared by incubating them with $200 \mu \mathrm{Ci}$ of $\mathrm{Na}_{2}{ }^{51} \mathrm{CrO}_{4}$ (BRIT, Bombay) for $60 \mathrm{~min}$ and subsequent washing to remove free ${ }^{51} \mathrm{Cr}$. Virus infection of target cells was carried out as mentioned earlier (Murali-Krishna et al 1994). At the end of the incubation period, an aliquot of cell-free supernatant was removed and ${ }^{51} \mathrm{Cr}$ released was counted in a LKB gamma counter. Triplicates and quadruplicates were routinely included in all assays and the \% lysis of target cells was calculated as [(cpm released in the presence of effector cells-cpm released due to spontaneous leak)/(total cpm released by $0 \cdot 1 \%$ Triton $\mathrm{X}-100-\mathrm{cpm}$ released due to spontaneous leak)] $\times 100$.

\subsection{Adoptive transfer of anti-JEV effectors}

Viable effector cells were isolated by layering the effectors generated on ficoll hypaque (Sigma, density 1119). Dead cells and RBC were removed, washed thrice with medium plus $0 \cdot 2 \%$ FBS and were resuspended in 5\% FBS containing RPMI 1640 medium before injection. Effector cells were injected intracerebrally (ic) along with $10 \times \mathrm{LD}_{50}$ $1 \mathrm{EV}$ in a total volume of $30 \mu 1$ using a sterile 26 gauge needle. Controls included injection with $1 \mathrm{EV}$ only, medium plus $5 \% \mathrm{FBS}$ or $1 \mathrm{EV}$ plus naive mouse spleen cells.

\subsection{Immunochemical localization of viral antigen in the brain tissue of mice}

The brains removed from the skull, in both the groups of animals were left in Zamboni's fixative (containing saturated picric acid solution in $4 \%$ paraformaldehyde) for $4 \mathrm{~h}$ at room temperature. They were washed in graded alcohols and left in $4 \%$ paraformaldehyde, after slicing into $1 \mathrm{~mm}$ thick blocks in the coronal plane until processed for paraffin embedding.

\section{$2.9 \quad$ Immunoperoxidase stain}

Six to eight micron thick paraffin sections from different anatomical areas of the brain were collected on polylysine coated slides. The endogenous peroxidase in the tissue 
section was quenched with $0 \cdot 3 \% \mathrm{H}_{2} \mathrm{O}_{2}$ in methanol and the nonspecific immune binding sites were blocked with $3 \%$ solution of defatted milk powder in PBS.JEV specific hyperimmune mouse ascitic fluid (gift obtained from National Institute of Virology, Pune) was the source of primary antibody. The sections were incubated in primary antibody $(1: 100)$ at $4^{\circ} \mathrm{C}$ overnight. After three rinses in PBS, the sections were incubated for $1 \mathrm{~h}$ in biotinylated goat antimouse $\operatorname{IgG}(1: 1000$, Sigma, USA). After further washing, the sections were incubated in freshly prepared streptavidin peroxidase complex (1:1000, Dakopats, USA) for $1 \mathrm{~h}$. All the dilutions of the primary and secondary antibody as well as streptavidin were made in $50 \mathrm{mM}$ PBS containing $0.05 \%$ Tween-20 (PBS-T) and 1\% defatted milk powder After washing with PBS-T the immune reaction was visualized by reacting the sections with diaminobenzidine tetrahydrochloride (DAB: $50 \mathrm{mg} \%$ Tris $\mathrm{HCl}$ buffer, $\mathrm{pH} 7 \cdot 5)$ and $\mathrm{H}_{2} \mathrm{O}_{2},(10 \mu \mathrm{l} / 10 \mathrm{ml})$. The sections were counterstained with Mayer's heamatoxylin. Appropriate negative and positive controls were incorporated All sections were blind-coded and examined by two investigators. The sections were read with special reference to the density of labelling and the topographic distribution of antigen bearing neurons in the unprotectted and protected mice.

\subsection{Statistical analysis}

Data representing survival curves were statistically analysed by the "G" test as recommended by Sokal and Rolf (1981) while viral titres were analysed by Students ' $t$ ' test (Sokal and Rolf 1981).

\section{Results}

\subsection{Virus clearance in adoptively transferred mice}

We have shown previously that anti-JEV CTL generated using the described protocol were Lyt $2 \cdot 2^{+}$and specifically recognized JEV in a $\mathrm{H}-2$ restricted manner. Neither JEV infected allogeneic nor infected xenogeneic PS cells used for immunization were lysed. These anti-JEV CTL also block the replication of virus in infected cells in vitro, (Murali-Krishna et al 1994). Virus titres were hence examined in the brains of normal and adoptively transferred mice in order to determine if virus was being cleared in vivo. After ic challenge with virus, three mice from each group were sacrificed and different organs were dissected out 4, 5 and 6 days postchallenge. They were homogenized and examined for infectious virus titres as described. As shown in table 1, detectable viral titres were not found in the brains of adoptively transferred mice while virus replicated to high titres in the brains of normal animals challenged Bic with $10 \times \mathrm{LD}_{50}$ of JEV. Viral titres were below detectable limits in organs like spleen and kidney of normal and adoptively transferred mice.

The specificity of anti-JEV effector mediated protection is shown in table 2.Neither anti $\mathrm{H}-2^{\mathrm{b}}$ effector cells nor naive splenocytes obtained from Balb/c mice were able to protect Balb/c mice against lethal Bic challenge with JEV. However, complete protection was seen when virus-specific effectors were injected ic along with JEV. In addition it was observed that anti-JEV effectors raised in Balb/c mice did not protect C57BL/6 mice against lethal ic challenge with JEV. 
Table 1. Virus titre in protected mice

\begin{tabular}{lcl}
\hline $\begin{array}{l}\text { ic injection } \\
\text { (day after challenge) }^{a}\end{array}$ & $\log _{10}$ pfu/brain $^{b}$ & Protection \\
\hline JEV & & \\
Brain & & \\
( 4 days) & $4 \cdot 14 \pm 0 \cdot 17^{c}$ & Not Protected \\
( 5 days) & $5 \cdot 08 \pm 0 \cdot 81$ & Not Protected \\
(6 days) & $5 \cdot 99 \pm 0 \cdot 39$ & Not Protected \\
Spleen & & \\
(6 days) & $<2$ & \\
Kidney & & \\
(6 days) & $<2$ & \\
JEV + anti-JEV effectors & & \\
(5 days) & & Protected \\
Brain & $<2$ & Protected \\
Spleen & $<2$ & \\
Kidney & $<2$ & \\
\hline
\end{tabular}

${ }^{a}$ Adult Balb/c mice (90-100 day old) were injected ic with $10 \times \mathrm{LD}_{50}$ along with or without $8 \times 10^{6}$ anti JEV effectors.

${ }^{b}$ Five days after IEV challenge/adoptive transfer, 3 mice from each group were sacrificed and the viral titre was examined in their brains.

${ }^{c}$ Differences of viral titres between this group and the protected group was statistically significant $(P<0.005)$.

Table 2. Specificity of anti-IEV effector mediated protection

\begin{tabular}{|c|c|c|}
\hline ic injection ${ }^{a}$ & Recipient & $\%$ Survival (dead/total $)^{d}$ \\
\hline JEV alone & $\mathrm{Balb} / \mathrm{c}$ & $0(10 / 10)$ \\
\hline $\mathrm{JEV}+$ anti-JEV effectors ${ }^{b}$ & -do- & $66(4 / 12)$ \\
\hline Anti-JEV effectors alone & -do- & $100(0 / 4)$ \\
\hline Anti $\mathrm{H}-2^{b}$ effectors alone & -do- & $83(1 / 6)$ \\
\hline $\mathrm{JEV}+$ anti $\mathrm{H}-2^{b}$ effectors ${ }^{c}$ & -do- & $0(8 / 8)$ \\
\hline $\mathrm{JEV}+$ splenocytes ${ }^{e}$ & -do- & $0(4 / 4)$ \\
\hline JEV alone & $\mathrm{C} 57 \mathrm{BL} / 6$ & $0(4 / 4)$ \\
\hline $\mathrm{JEV}+$ anti-JEV effectors & -do- & $0(5 / 5)$ \\
\hline
\end{tabular}

${ }^{a}$ Mice were injected ic with $10 \times \mathrm{LD}_{50} \mathrm{JEV}$ alone or in combination with $16 \times 10^{6}$ anti-IEV effector cells.

${ }^{\mathrm{b}}$ Anti-JEV effector cells were generated in Balb/c mice as described in optimal immunization and in vitro stimulation protocol.

${ }^{c}$ Anti $\mathrm{H}-2^{b}$ effector cells were generated in Balb/c mice by in vivo immunization and in vitro stimulation with allogeneic C57BL/6 mouse spleen cells. These CTL exhibited $64 \%$ lysis on ${ }^{51}$ Cr-labelled EL-4 $\left(\mathrm{H}-2^{b}\right)$ targets at an E : T ratio of $80: 1$. ${ }^{d}$ Survival of the mice was monitored for a period of 30 days after challenge /adoptive transfer

${ }^{e}$ Splenocytes were obtained from naive unimmunized Balb/c mice.

\subsection{Viral antigen detection by immunohistochemistry}

On routine histological evaluation, a striking feature observed in both the groups was the meningeal infiltrate of lymphocytes and antigen bearing histiocytes though 

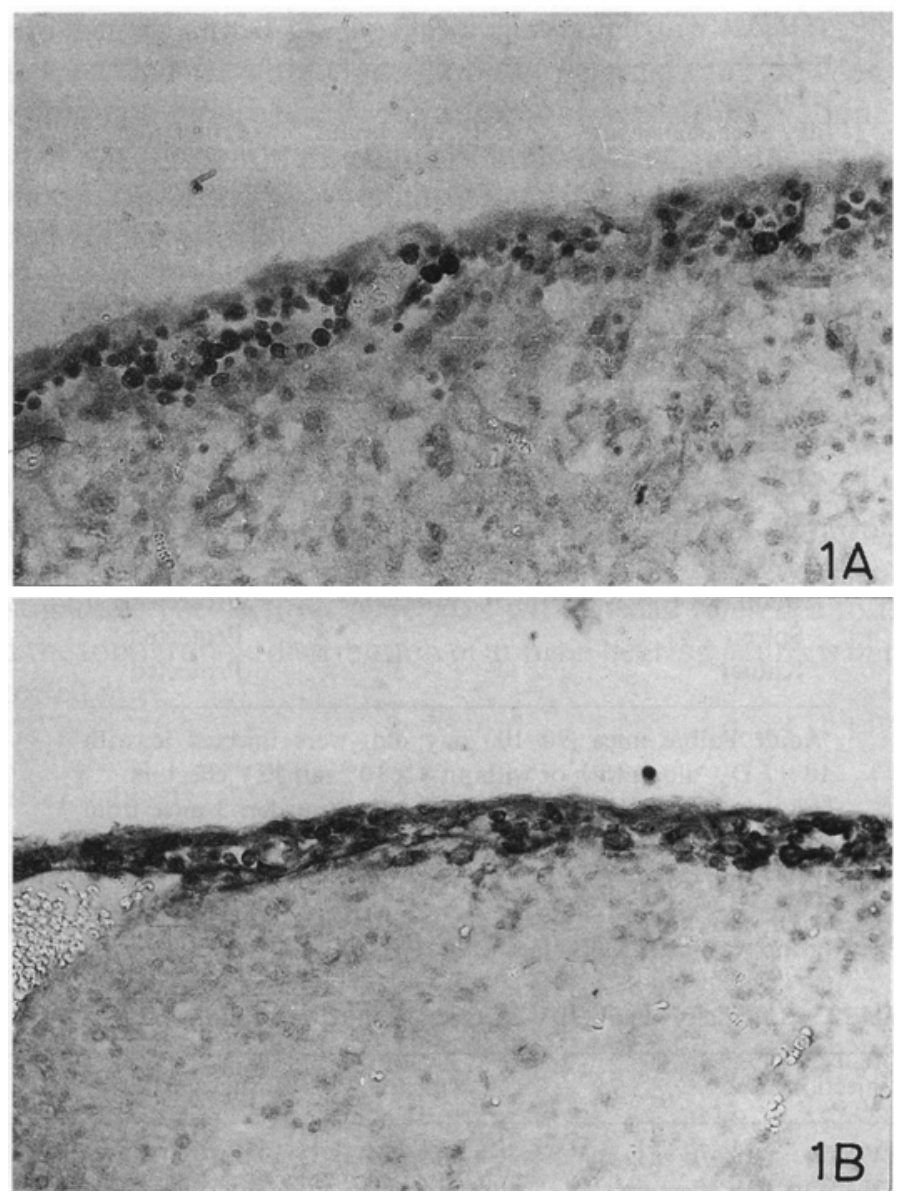

Figure 1. (A) Meningeal inflammatory infiltrate in the unprotected mouse brain containing many dark Japanese encephalitis viral antigen bearing histiocytes (X 200). (B) Meningeal inflammatory infiltrate in the protected mouse brain, shown antigen containing histiocytes, similar, but less than in A (X 200).

marginally less in the protected group (figure 1). These infiltrates, focally were found to spread along the perivascular space into the brain parenchyma. In the unprotected group of mice, microglial response was diffuse in different anatomical areas, with an occasional focus neuronophagia in the cerebral cortex, unlike the protected group where it was mild and sparse. In the brain parenchyma of the control unprotected group, immunohistochernically antigen labelled neurons were numerous in the cerebral cortex (figure 2A, B), hippocampus, large neurons of the reticular formation in the brain stem and various cranial nerve nuclei. In addition to intraneuronal antigen, in the hippocampus focal extra cellular antigen could be identified, representing the ruptured neuronal cell soma, following viral infection. In the cerebellum, Purkinje cells and small neurons of the granular layer were variably labelled. A few of the white fibre tracts were lightly immunolabelled indicating antigen spread along the axons. 

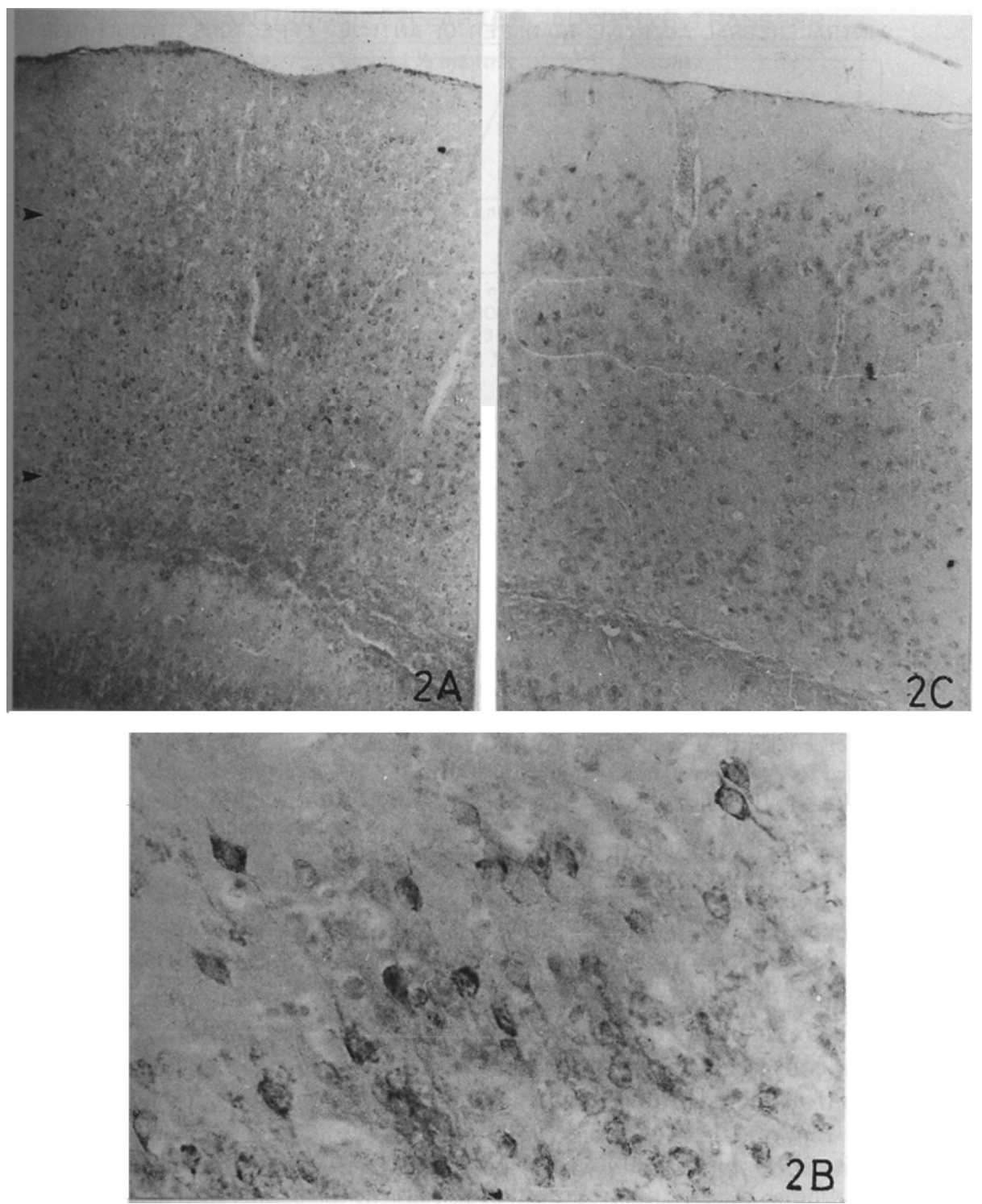

Figure 2. (A) Cerebral cortex of the unprotected mouse shows many dark antigen bearing neurons in the lower layers (arrows to mark border) (X 80). (B) Higher magnification of unprotected mouse cerebral cortex showing numerous neurons containing intracytoplasmic viral antigen, in some spreading along the dendritic processes (X 300). (C) Cerebral cortex of the protected mouse brain showing lack of antigen bearing neurons (X 80).

In the group of animals protected by adoptively transferring the sensitized lymphocytes, the antigen bearing neurons were sparse and randomly distributed (figure 2C). While the cerebral cortex and hippocampus had a rare labelled neuron, a few small groups of neurons containing intracytoplasmic antigen were found in brainstem, while none were found in the cerebellum. 
INTRACEREBRAL ADOPTIVE TRANSFER OF ANTI-JEV EFFECTORS TO ADULT MICE

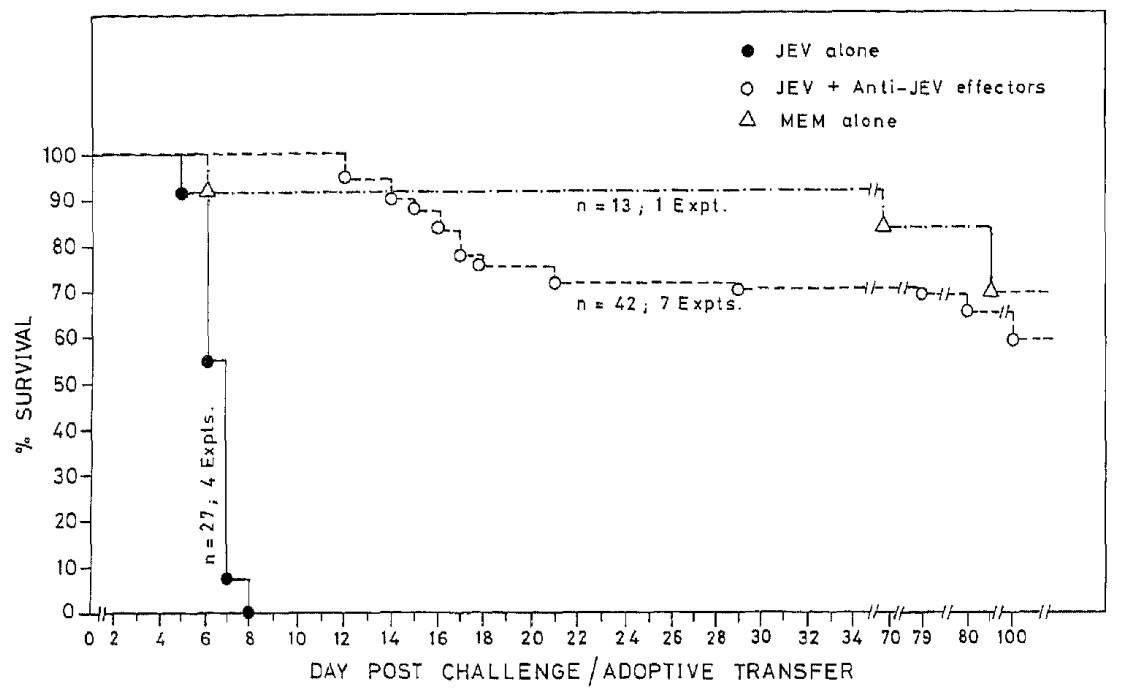

Figure 3. Long term protection of mice with adoptively transferred anti-JEV effectors. Anti-JEV effectors were generated and isolated by ficoll hypaque centrifugation as described in $\$ 2 \cdot$ Adult male Balb/c mice were injected ic with $8 \times 10^{6}$ effectors along with $10 \times \mathrm{LD}_{50}(\mathrm{O})$ $\left(n=42\right.$; data pooled from 7 experiments), with $10 \times \operatorname{LD}_{50} \mathrm{JEV}$ alone $(\bullet)(n=27$; data pooled from 4 experiments) or with $30 \mu 1$ of RPMI:1640 containing 5\% FBS $(\Delta)(n=13)$ and monitored for mortality over a period of 100 days Anti-JEV effectors exhibited $52-71 \%$ specific lysis on JEV-infected targets at an E:T ratio of 80:1 before adoptive transfer-Values are expressed as cumulative percentage of surviving mice. Statistical significance ' $P$ ' value between $(\bullet)$ and $(\mathrm{O})$ was $<0001,(\mathrm{O})$ and $(\Delta)$ was $<0.05$.

\subsection{Protective ability of adoptively transferred anti-JEV effectors}

Adoptive transfer experiments were carried out to demonstrate the protective ability of anti-JEY effectors generated. Anti-JEV effectors were injected intracerebrally along with simultaneous lethal challenge by $10 \times \mathrm{LD}_{50} 1 \mathrm{EY}$ as given in $\S 2$ in a total volume of $30 \mu \mathrm{l}$. Controls included mice challenged with $10 \mathrm{x} \mathrm{LD}_{50} \mathrm{JEV}$ ic and injection of $30 \mu 1$ RPMI 1640 medium containing 5\% FBS ic. While mice challenged with JEV alone exhibited $100 \%$ mortality within 7-9 days, medium injected mice did not die within this period suggesting that death was not due to the Bic injection per se. When the mortality of adoptively transferred mice was recorded over a period of 100 days, all the mice survived until 12 days after $1 \mathrm{EV}$ challenge but $55 \%$ survived beyond 100 days. It must be mentioned that $47 \%$ survived for as long as 7 months after adoptive transfer (figure 3).

\subsection{Recovery of CTL activity in naive adoptively transferred mice}

Cytotoxic T-cells have been recovered from the brains of mice infected with WNY (Liu et al 1989). However, our preliminary attempts to demonstrate anti-JEV CTL in the brains of immunized animals were unsuccessful (data not shown). Hence we examined if CTL could be recovered from the spleens of mice undergoing Bic adoptive transfer. Mice 
ANTIVIRAL CTL AFTER ADOPTIVE TRANSFER

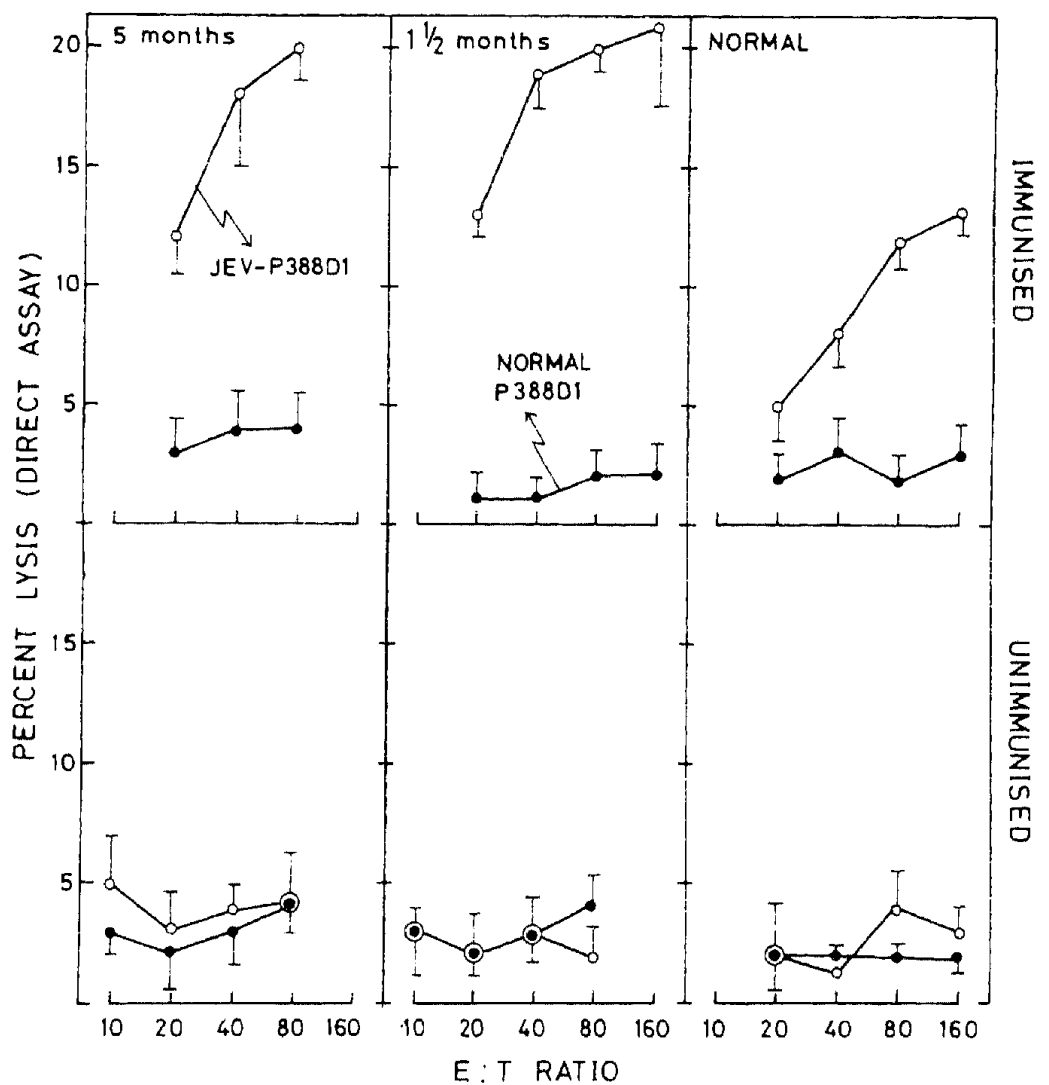

Figure 4. Anti-JEV effector activity in adoptively transferred mice. Adult Balb/c mice that had been protected from lethal intracerebral JEV challenge by adoptively transferred CTL were primed in vivo as described in $\S 2$. After sacrifice, spleen cells were assayed on JEVinfected $(\mathrm{O})$ and uninfected $(\bullet)$ P388D1 targets as given in $\S 2$. without restimulation in vitro. Controls included adoptively transferred mice that were not primed in vivo. Values are expressed as mean percentage lysis \pm S D. Spontaneous release of ${ }^{51} \mathrm{Cr}$ was $16 \%$ and $30 \%$ for normal and infected P388D1 respectively.

that were protected from JEV-induced death by adoptive transfer of anti-JEV effectors were immunized at different times after the first JEV challenge. The optimal immunization protocol was followed. The splenocytes obtained were assayed directly on relevant syngeneic ${ }^{51} \mathrm{Cr}$ labelled targets without secondary in vitro restimulation. Controls included adoptively transferred mice that were not subjected to the optimal immunization protocol. As shown in figure 4, using the direct assay, no significant differences were seen in lysis of syngeneic infected or uninfected targets by splenocytes obtained from naive adoptively transferred mice. However, splenocytes obtained from adoptively transferred mice that were immunized specifically lysed syngeneic infected P388D1 cells to a greater degree relative to splenocytes from naive adoptively transferred mice. Splenocytes were obtained from immunized mice that were protected for 45 and 150 days after is lethal JEV challenge. They exhibited $18 \%$ and $16 \%$ lysis on infected targets at E : T of $40: 1$ respectively. Splenocytes were also obtained from control mice that were not adoptively transferred but were only subjected to the optimal immunization protocol. Killing obtained with these effectors was only $8 \%$ (figure 4 ). 
In a separate experiment, splenocytes were also obtained from adoptively transferred mice that were subjected to the optimal immunization protocol. However, anti viral CTL were generated by restimulating the splenocytes obtained with syngeneic infected cells in vitro. Controls included adoptively transferred mice that were not immunized optimally. Splenocytes were also obtained from normal mice that were not adoptively transferred with anti-JEV effectors. All effectors generated after secondary in vitro restimulation were assayed against ${ }^{51} \mathrm{Cr}$-labelled infected and uninfected P338D1 targets. As shown in figure 5, effectors generated from normal mice exhibited $49 \%$ and $7 \%$ lysis on infected and uninfected P333D1 cells respectively. However, if the effectors were generated from mice that were protected for 45 days by adoptive transfer, the lysis on infected cells was much higher (71\%). No specific lysis of infected cells was evident when effectors were generated by restimulation in vitro of splenocytes obtained from normal unimmunized animals. Effectors were also generated from unimmunized but adoptively transferred animals. Infected targets were significantly lysed by these effectors at an $\mathrm{E}: \mathrm{T}$ ratio of $20: 1$ (figure 5).

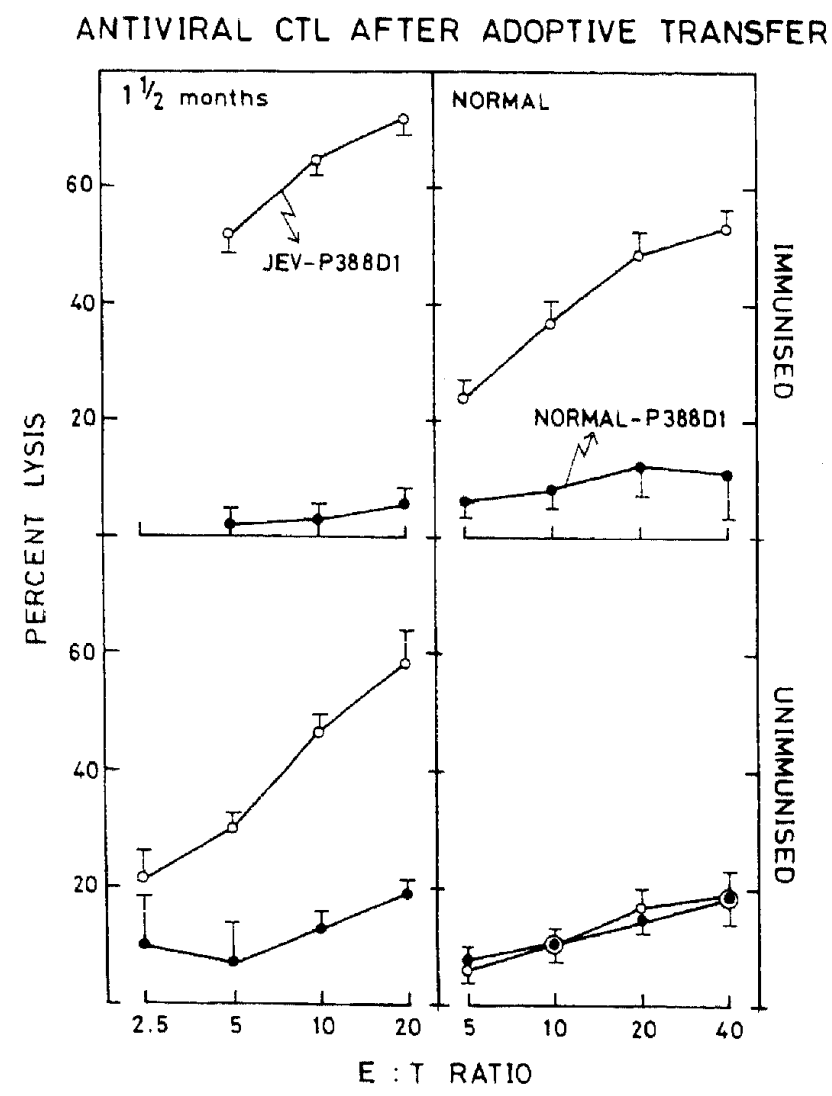

Figure 5. Generation of anti-JEV effectors in adoptively transferred mice. Adult Balb/c mice that had been protected from lethal intracerebral $3 \mathrm{EV}$ challenge by adoptively transferred CTL were primed in vivo as described in $\S 2$. After sacrifice, spleen cells were restimulated in vitro with 3EV-infected P338D1 and the CTL-generated were assayed on JEV-infected (O) and uninfected (•) P388D1 targets as given in $\$ 2$. Controls included adoptively transferred mice that were not primed in vivo. Values are expressed as mean percentage lysis $\pm \mathrm{S} D$. Spontaneous release of ${ }_{51} \mathrm{Cr}$ was $16 \%$ and $30 \%$ for normal and infected P338D 1 respectively. 


\section{Discussion}

A variety of humoral and cellular effector mechanisms mediate host defense against viral infections (Gajdosova et al 1931; Allan and Doherty 1986). Similarly, stimulations of the immune system have been seen both in humans and mice in response to JEV infection (Thakare et al 1991; Desai et al 1995) as well as other flaviviruses. Human $\mathrm{CD}^{+}$and $\mathrm{CD}^{+}$cytotoxic $\mathrm{T}$-cell responses have been reported to dengue virusinfected cells. Adoptive transfer of immune T-cells from $1 \mathrm{EV}$ immunized mice to normal mice confers protection against disease (Mathur et al 1933) suggesting the involvement of a T-cell component in protection. However, the status of cell mediated immunity during JEV infection is still not completely understood. We have previously shown that CTL can be raised to JEV in Balb/c mice. These CTL were also shown to protect adult but not newborn mice against lethal intracerebral challenge with JEV (Murali-Krishna et al 1996). In this report we demonstrate by immunohistochemistry that viral antigen is cleared from the brains of virus injected mice by intracerebral adoptive transfer of virus-specific CTL. We further show that increased CTL activity can be recovered from the spleens of adoptively transferred mice.

The function of T-cells in virus clearance and immunopathology has also been analysed in many virus infections (Hill et al 1995; Klavinski et al 1939). Adoptively transferred CTL have been shown to confer protection in several viral infections. In a mouse model, CTL specific for LCMV did not lyse infected neurons or cause immunopathologic injury. However, intracerebral transfer of the same CTL clones to acutely infected mice resulted in death within 7 to 12 days (Byrne and Oldstone 1984). We show that adoptively transferred effectors confer protection to adult Balb/c mice against intracerebral challenge with $10 \times \mathrm{LD}_{50} 1 \mathrm{EV}$. Protection lasted as long as 100 days after virus challenge. Protection of adult mice was not observed when effectors were transferred by intravenous or intraperitoneal routes (Murali-Krishna et al 1996). A similar observation requiring the transfer of CTL by is route for complete clearance of LCMV from the CNS has been reported (Klavinski et al 1989). Our experiments demonstrate that adoptive transfer of effectors resulted in complete clearance of virus from the brains of mice lethally injected with JEV. This was confirmed by viral plaque assays as well as immunohistochemical assays. In view of the possibility that the adoptively transferred CTL could also have migrated out from the brain, we examined the spleens for the presence of cytotoxic T-cells. Indeed, CTL generation was found to be higher when splenocytes from unimmunized but adoptively transferred mice were restimulated in vitro with syngeneic JEV-infected cells (figure 5). It must be noted that significant levels of anti-JEV CTL activity could not be generated either by in vivo immunization alone or in vitro stimulation alone (Murali-Krishna et al 1994). It is unlikely that the CTL activity generated in adoptively transferred mice is due to stimulation by injected virus in view of the significant inhibition of virus growth observed in the brains of protected mice using viral and immunohistochemical assays. Therefore, we interpret that the CTL activity generated was due to the presence of donor derived CTL that have migrated to the spleen from the brain. This conclusion is further supported by the observation of detectable CTL activity using direct CTL assays (figure 4) as well as increased CTL generation in immunized and adoptively transferred mice relative to normal mice that had not undergone adoptive transfer (figure 5). In the case of Theiler's murine encephalitis infection, demyelination is partly mediated by CD8 positive $\mathrm{H}-2$ restricted T-cells that are found in the CNS of 
susceptible mice (Lindsley et al 1991). Our immunohistochemical analysis of the brains from JEV protected mice did not reveal signs of immunopathology even though anti-JEV effectors were delivered ic. Thus it would be important not only to analyse the mechanisms of JEV clearance in such mice but also to investigate the mechanisms of effector migration from the brains of adoptively transferred and protected mice.

\section{Acknowledgements}

This work was partly supported by a grant from the Department of Science and Technology, New Delhi· KMK and BRR were senior research fellowship holders from the University Grants Commission, New Delhi during the course of this investigation. The authors wish to thank Dr N V Joshi (Center for Ecological Sciences) for help in statistical analysis.

\section{References}

Allan J E and Doherty P C 1986 Stimulation of helper/delayed type hypersensitivity T cells by flavivirus infection: determination by macrophage procoagulant assay; J. Gen·Virol. 67 39-46

Byrne J A and Oldstone M B A 1984 Biology of cloned cytotoxic T lymphocytes specific for lymphocytic choriomeningitis virus: clearance of virus in vivo; J· Virol. 51 682-686

Chambers T J, Hahn C S, Galler R and Rice C M 1990 Flavivirus genome organization, expression, and replication; Annu Rev Microbiol. 44 649-688

Cossins J, Gould K G, Smith M, Driscoll P and Brownlee G G 1993 Precise prediction of a $\mathrm{K}^{k}$-restricted cytotoxic T cell epitope in the NS 1 protein of influenza virus using an MHC allele specific motif; Virology $193289-295$

Desai A, Ravi V, Chandrarnukhi A and Gowrie Devi M 1995 Protective response of human peripheral blood mononuclear cells to Japanese encephalitis virus; Microbiol Immunol. 39 269-273

Gajdosova E, Oravec C and Mayer V 1981 Cell mediated immunity to flavivirus infections 1 . Induction of cytotoxic T lymphocytes in mice by an attenuated virus from the tick-borne encephalitis complex and its group reactive character; Acta Virol. 25 10-18

Gould E A and Clegg J C S 1985 Growth, titration and purification of alphaviruses and flaviviruses; In Virology: A practical approach, (ed.) E W J Mahy (Oxford: IRL Press) pp 43-74

Hill A B, Lobigs M, Blander R V, Kulkarni A and Mullbacher A 1995 The cellular immune responses to flaviviruses; in Viruses and cellular immune response (ed·) D B Thomas (New York: Marcel Dekker) pp 363-388

Joly E, Mucke L and Oldstone M B A 1991 Viral persistence in neurons explained by lack of major histocompatibility class I expression; Scie nce 253 1283-1285

Kawano H, Mifune K, Ohuchi M, Mannen K, Cho S, Hiramatsu K and Shichijo A 1990 Protection against rabies in mice by a cytotoxic $\mathrm{T}$ cell clone recognising the glycoprotein of rabies virus; $J \cdot G e n \cdot V i r o l .71$ 281-287

Kedarnath N, Dayaraj C, Sathe P S, Gadkari D A, Dandavate C N Goverdhan M K and Ghosh S N 1986 Monoclonal antibodies against Japanese encephalitis virus; Indian J Med·Res·84 125-133

Kimura-Kuroda J and Yasui K 1988 Protection of mice against Japanese encephalitis virus by passive administration with monoclonal antibodies; J. Immunol.144 3606-3610

Klavinski L S, Tishon A and Oldstone M B A 1989 Efficiency and effectiveness of cloned virus-specific cytotoxic T lymphocytes in vivo; J. Immunol. 143 2013-2016

Lad V J, Gupta A K, Goverdhan M K, Ayachit V L Rodriguez J J and Hungund L V 1993 Susceptibility of BL6 Nude (congenitally athymic) mice to Japanese encephalitis virus by the peripheral route; Acta Virol. 37 232-240

Lindsley M D, Thiemann R and Rodriguez M 1991 Cytotoxic T cells isolated from the central nervous system of mice infected with Theiler's virus; J.Virol. 65 6612-6620

Liu Y, Blanden R V and Mullbacher A 1989 Identification of cytotoxic T lymphocytes in West Nile virus infected murine central nervous system; J· Gen. Virol. 70 565-573 
Mathur A, Arora K L and Chaturvedi U C 1983 Host defense mechanisms against Japanese encephalitis virus infection in mice; J. Gen. Viral. 64 805-811

Monath T P 1986 Pathobiology of flaviviruses; in The Togaviridae and flaviviruses, (eds·) S Schlesinger and M J Schlesinger (New York, London: Plenum Press) pp 375-440

Murali-Krishna K, Ravi V and Manjunath R 1994 Cytotoxic T lymphocytes raised against Japanese encephalitis virus: effector cell phenotype, target specificity and in vitro virus clearance; J. Gen. Viral. 75 799-807

Murali-Krishna K, Ravi V and Manjunath R 1995 Japanese encephalitis virus infection of mouse cell lines: Ability to prime mice for generation of virus specific cytotoxic $\mathrm{T}$ lymphocytes and differences in CTL recognisable viral determinants; Arch· Virol. 140 127-143

Murali-Krishna K, Ravi V and Manjunath R 1996 Protection of adult but not newborn mice against intracerebral lethal challenge with Japanese Encephalitis Virus by adoptively transferred Cytotoxic T Lymphocytes: Requirement for L3T4 cells; J. Gen. Viral. 77 705-711

Oyanagi S, Fusahiro I and Ross E R 1969 Electron microscopic observations in mice infected with Japanese encephalitis virus; Acta Iveuropathol. 13 169-181

Rothman A L, Kurane I, Lai C -J, Bray M, Falgout B, Men R and Ennis F A 1993 Dengue virus protein recognition by virus-specific murine $\mathrm{CD} 8^{+}$cytotoxic T lymphocytes; J V Virol. 67 801-806

Sokal R R and Rolf F J 1981 Principles of statistics in biological research 2nd edition (New York: W H Freeman)

Thakare J P, Gore M M, Risbud A R, Banerjee K and Ghosh S N 1991 Detection of virus specific IgG subclasses in Japanese encephalitis patients; Indian J· Med·Res. A93 271276

Westaway E G, Brinton M A, Gaidamovich S Y, Horzinek M C, Igarashi A, Kaariaineen L, Lvov D K, Porterfield J S, Russel P K and Trent D W 1985 flaviviruses; Intervirology 24 183-192 